\title{
Direct Imaging of Metal Additives on Au Nanocube Surface Using 4D-STEM
}

\author{
Weilun Li ${ }^{1}$, Bryan D. Esser ${ }^{1}$, Anchal Yadav ${ }^{2}$, Alison M. Funston ${ }^{2,3}$, S.D. Findlay ${ }^{4}$, Changlin Zheng ${ }^{5,6}$, Joanne \\ Etheridge ${ }^{1,5}$
}

\author{
${ }^{1}$ Department of Materials Science and Engineering, ${ }^{2}$ School of Chemistry, ${ }^{3}$ ARC Centre of Excellent in Exciton Science, ${ }^{4}$ School of \\ Physics and Astronomy, ${ }^{5}$ Monash Centre for Electron Microscopy, Monash University, Victoria 3800, Australia, ${ }^{6}$ State Key \\ Laboratory of Surface Physics and Department of Physics, Fudan University, Shanghai 200433, China \\ weilun.li@monash.edu
}

Recent developments in the ability to control the shape of metal nanocrystals using wet chemistry synthesis techniques have drawn significant attention for potential applications in plasmonics, photonics and catalysis. It is conjectured that shape control can be achieved by controlling the nanocrystal surface structure, primarily using surfactants and metal additives. For example, various shapes of Au nanoparticles, including rods, cubes, decahedra and octahedra, can be grown selectively using a trace amount of $\mathrm{Ag}$ [1] or $\mathrm{Cu}$ [2] additives. Understanding the underlying mechanisms of shape control by metal additives is therefore vital for the further engineering of nanocrystals. However, the establishment of atomic structure models of metal additives on the nanocrystal surface, that are just a few atoms wide, is still challenging.

For this aim, there are three essential requirements of the characterisation technique: (1) sufficient chemical sensitivity to distinguish surface additives; (2) spatial resolution at the atomic scale; (3) avoidance of damage to the surface structure from the probe.

Scanning transmission electron microscopy (STEM) has been widely used as a powerful means of resolving atomistic structures of nanocrystals. In this study, we studied the structure of $\mathrm{Cu}$ additives on the surface of $\mathrm{Au}$ nanocubes using conventional energy dispersive X-ray spectroscopy STEM (EDX-STEM) and high-angle annular dark filed STEM (HAADF-STEM), as well as fourdimensional STEM (4D-STEM) tuned specifically for the detection of surface adatoms.

EDX-STEM mapping reveals the presence of $\mathrm{Cu}$ on the $\{100\}$ surfaces of Au nanocubes. However, the high electron dose required to achieve meaningful statistics can damage the structure during acquisition so a quantitative, high resolution analysis of an undamaged surface is not possible.

HAADF-STEM collects electrons at high angles by an annular detector and presents intensity sensitive to the atomic number (Z), however, it is also sensitive to other material parameters such as thickness and local environments (such as vacuum) $[3,4]$. We observe the intensity to drop at the nanoparticle atomic surface layer in the HAADF-STEM image but cannot distinguish whether this is due exclusively to the presence of (lower $\mathrm{Z}$ ) $\mathrm{Cu}$ atoms or just fewer atoms on the surface layer. Furthermore, this is a dose-inefficient technique, using only those electrons scattered to high angles, and surface modification can be observed during acquisition.

4D-STEM using a fast pixelated detector records the full diffraction patterns at each probe position during the STEM experiment. The collected 4D datasets enable us to investigate features in diffraction patterns that are specifically related to different material parameters [4]. This method has the advantages of a lower dose than EDX-STEM and access to much more specimen information than HAADF-STEM. In this work, we developed an iterative method by starting with an ideal nanocube model with uniform thickness and with/without surface $\mathrm{Cu}$ layers. Dynamic diffraction conditions and scattering angles sensitive to the presence of $\mathrm{Cu}$ additives were identified based on the comprehensive dynamical scattering 4D-STEM simulations of this initial model. We then collected experimental 4D-STEM datasets using our optimised imaging conditions, from which $\mathrm{Cu}$ adatoms on the surface were evident with excellent contrast. In the next step, a matching of experimental diffraction patterns with simulated diffraction patterns was conducted to determine the realistic surface thickness profile. These allowed us to further refine the nanocube model using the observed surface structure of $\mathrm{Cu}$ adatoms and fitted thickness profile for the ultimate 4D-STEM simulations. Excellent agreement was achieved in both qualitative and quantitative comparisons between 4D-STEM simulations and 4D-STEM experiments over various imaging models (which each correspond to different diffraction physics). This suggests that the refined nanocube surface model represents the actual structure of $\mathrm{Cu}$ additives on the surface of Au nanocubes. This provides an approach for identifying the type and arrangement of the critical surface atoms that play an important role in controlling the growth and shape of nanoparticles.

[1] Personick, Michelle L., et al. Nano letters 11.8 (2011): 3394-3398. [2] Sun, Jianhua, et al. Crystal Growth and Design 8.3 (2008): 906-910.

[3] LeBeau, James M., et al. 100.20 (2008): 206101. [4] Cowley, John. M. Surface Science 114.2-3 (1982): 587-606.

[4] Ophus, Colin. Microscopy and Microanalysis 25.3 (2019): 563-582.

Keywords: Metal nanocrystals; Metal additives; Surface structure; 4D-STEM; STEM simulation

This work was carried out on the microscopes at the Monash Centre for Electron Microscopy funded by ARC grant number (LE0454166). This work was supported by ARC funding Discovery Project number DP160104679. 